\title{
Comparison of Image Classification Techniques and its Applications
}

\author{
Kushalatha. M. R. \\ Assistant Professor, \\ Department of ECE, \\ Nitte Meenakshi Institute of Technology, \\ Bangalore, India \\ Kushalatha.mr@nmit.ac.in
}

\author{
Dr. H. S. Prashantha. \\ Professor, \\ Department of ECE, \\ Nitte Meenakshi Institute of Technology, \\ Bangalore, India \\ prashantha.hs@nmit.ac.in
}

\author{
Beena. R. Shetty \\ Assistant professor, \\ Department of ECE, \\ Nitte Meenakshi Institute of Technology, \\ Bangalore, India \\ beena.s.rai@nmit.ac.in
}

\begin{abstract}
Image classification has become a hot topic in the field of remote sensing. In general, the complex characteristics of multispectral data make the accurate classification of such data challenging for traditional machine learning methods. In addition, multispectral imaging often deals with an inherently nonlinear relation between the captured spectral information and the corresponding materials. In this paper, we propose a novel classification method for multispectral imagery, named as support vector machine (SVM). Pixel multispectral imagery can be represented by amplitude, phase and residual in frequency. Its applicability and effects are assessed by the experiment using data set, in which CNN, and KNN based feature extraction methods are adopted for comparison, aiming to evaluate the performance of the proposed method. Experimental results illustrate that the proposed model gains the highest classification accuracy. Comparison of various performance parameters showed that KNN works better than SVM.
\end{abstract}

Keywords: Multispectral $l$ imaging, Support Vector Machines, K-Nearest Neighbour, Classification accuracy

\section{INTRODUCTION}

SVM is a machine learning algorithm based on the linear discrimination for binary classification problems. The major advantage of SVM is that only a few training samples are required for machine learning to establish a hyperplane to separate spectral classes. In SVM, the pixels that were placed at the edge of the class are the support vectors used for the feature training. If a pixel locates beyond the optimal margin, for example, the two black dots and the two white circles mixed in the opposite feature will be misclassified.

The researches carried out in last few decades extracted a lot amount of information with respect to spatial, temporal and spectral resolutions. The multispectral imaging carries a generous amount of spectral information, which introduced many newer applications, new technological challenges and advancement in newer domains of research [1]. The high spectral resolution made the multispectral imaging to be used in extraction of subtle objects, materials with and are widely used in applications like remote sensing, quantification [2], identification, and surveillance [3] and in precision urban agriculture [4]. The process of assigning the individual pixels to a definite group of classification classes (Trained or Untrained depending on Supervised or Unsupervised classification) is a tedious process in classification of images. The training samples available in a classification approach can be divided into mainly 2 categories, i.e., Supervised and Unsupervised classifiers. In the supervised approach the classification procedure is based on (classification of input data) already available samples known as training samples where in unsupervised doesn't have such available samples for classification purpose. The multispectral classification has taken a large number of advances with respect to its classification efficiency; it still poses problems with respect to high dimensionality, unbalanced and limited number of training samples available, mixing of pixels and training samples [5]. Second problem is that; the classification efficiency reduces when a smaller number of samples is taken. The third problem arises with the curse of increment in dimensions of data and high redundancy between the features, which make the classification in its analysis stage. A large degree of literature surveys has been conducted as to overcome these problems, and to conduct Hyperspectral image classification in an effective way.

Linear SVM, S3VM, SVM Cubic Methods, Least Square SVM, Iterative SVM, Relevance SVM, Binary tree SVM, Probabilistic SVM are some of the variations of SVM algorithms and are the prominent methods which have been proposed in recent years for the classification of Multispectral data. Based on the techniques of utilising the training samples, image classification process is categorized as Supervised, Unsupervised and Semi-Supervised multispectral Image classification.

\section{SURVEY CARRIED OUT}

Chunyu $\mathrm{Hu}(2020)$ proposed a method which combined the fast guided filter and like neighborhood information of image classification (FGF - SVM - SNI) algorithm, using principal component analysis and fast orientation filter work together to extract spatial texture information, the filtered spatial texture information is linearly fused with the spectral information, after the space spectrum information is formed, it is classified by the support vector machine supported by the radial basis function. Then, a spectral pixel neighborhood spatial information method is designed to construct spatial correlation information to optimize the classification results.

The work proposed by Peter Burai focused on the main objective of applying machine learning methods using crown segments for image classification. A watershed segmentation algorithm was used to delineate individual crowns from a filtered CHM model. The image classification was applied on the original spectral bands and transformed (MNF) dataset. A binary tree SVM classifier was developed in accordance with the principle of SVM, based on the Jeffries-Matusita (JM) reparability measure of selected classes. The ABTSVM on MNF-transformed dataset provided more accurate results than applied multiclass SVM methods. The addition of crown segments resulted in an increase in classification accuracy of 14.51 percentage points over pixel-based classification alone. The results obtained showed that, Multi-class SVM 
classification on the originals data sets provided only poor accuracy (OA: 62.46\%, kappa: 0.48). To minimize the influence of sensor noise during image analysis and reduce redundant data, minimum noise fraction (MNF) transformation was used. It was found that the best SVM classification results in the cases of first 13 MNF bands. The addition of MNF bands couldn't increase significantly the accuracy of classification.

Abu Sayeed (2020) proposed a method where Principal Component Analysis (PCA) was used as to extracts feature however it depends on global variance. To overcome these limitations, a class-oriented subspace detection method is proposed which measures the relevancy of the selected subspace using Normalized Mutual Information (NMI) and Cross Cumulative Residual Entropy (CCRE). The application of NMI and CCRE over PCA images maximize the relevance as well as provided an uncorrelated subspace. Experimental analysis was performed to assess the effectiveness of the proposed method and the selected subspace was evaluated using the Support Vector Machine (SVM). The proposed method achieved $87.61 \%$ and $88.57 \%$ classification accuracy respectively.

Vineetha Menon proposed a method that made use of Composite Kernel support vector machine (SVM-CK) which was used to incorporate spatial-spectral information for enhanced supervised classification performance. It is experimentally demonstrated that proposed RP based NMF feature extraction approaches can perform other methods. This paper introduced a novel computationally fast and effective RP based feature extraction using NMF for DR and hyperspectral classification applications. It was experimentally validated that both the proposed RP based NMF feature extraction methods i.e., HM-NMF, and GM$\mathrm{NMF}$, can offer superior classification performance, even at lower dimensions compared to other methods in comparison. Especially, HMNMF had better information preservation even at far fewer dimensions. Both HM-NMF and GM-NMF proved to be computationally efficient. Hence, RP based DR methods serve as an excellent means for multispectral data analysis and alleviates the associated high computation and storage overhead.

Shengwei Zhong (2018) work developed a novel approach to multispectral image classification, referred to as iterative SVM (ISVM) based on feeding back Gaussian filtered-classification maps. By virtue of the designed iterative process, spatial classification information is iteratively captured and added into training samples to increase the classification results. Experimental results demonstrate that ISVM outperforms other spectral-spatial methods.

\section{A. Unsupervised Classification}

The main challenge posed by the classification approaches in Hyperspectral imaging is high dimensionality. As to overcome these curse varieties of feature extraction techniques are extensively made use of in classification process. The prominent features are only selected as to reduce the problem of dimensionality instead of selecting the entire features in an image. In case of unsupervised techniques, the automatic selections of group of pixels which carry similar information like means, deviations are done. The concept is based on type of algorithms used. Principal Component Analysis (PCA), Independent
Component Analysis (ICA) is some of the very famous classification techniques under Unsupervised Classification.

1) Principal Component Analysis (PCA): PCA is mainly used method in classification as it helps in dimensionality reduction. The dimensionality reduction is accomplished by reducing the number of variables while in the original dataset most of the information is still retained. The basis on which the PCA works is the existence of good correlation between Hyperspectral image bands. PCA mainly deals with the second order statistics and these statistics cannot describe the higher resolution data, which are mainly made use of in Hyperspectral imaging.

\section{B. Supervised Classification}

The supervised classification takes the data which is labelled in prior as to train the classifier. The advantage of supervised with respect to the unsupervised is that it can be applied to those images which contains rich spectral information A large number of supervised classification methods have been summarised in the literature, some of the prominent methods are maximum likelihood (ML), nearest neighbour classifier, decision trees, random forest, support vector machines (SVMs), Spectral Angle Mapper (SAM) etc.

1) Support Vector Machine (SVM): SVM is a very strong and prominent classification approach, which makes use of Kernel functions and works on the basis of theory of optimization. SVM is proved to a better technique of classification in environments where the image is represented in more number of spectral bands and availability of less number of training samples. Figure 1 shows a SVM model in multidimensional space which represents different classes in a Hyperplane. The Hyperplane is generated in an iterative manner as to reduce the error in classification. The maximum Marginal Hyperplane (MMH) is obtained by SVM by dividing the given datasets into variety of classes.

K- Nearest Neighbour Algorithm (KNN): The proposed method consists of the following steps. Initial step is to adapt a support vector machine (SVM) technique. The SVM helps to obtain classification probability maps which in turn help to reflect the probability with which each of the pixels of the HSI belong to the different classes of classification. The filtering process of $\mathrm{KNN}$ includes matching and non-local averaging of the neighbourhood pixels. Another important parameter used by KNN is Euclidian distance metric, which works as a distance metric when the data is homogeneous.

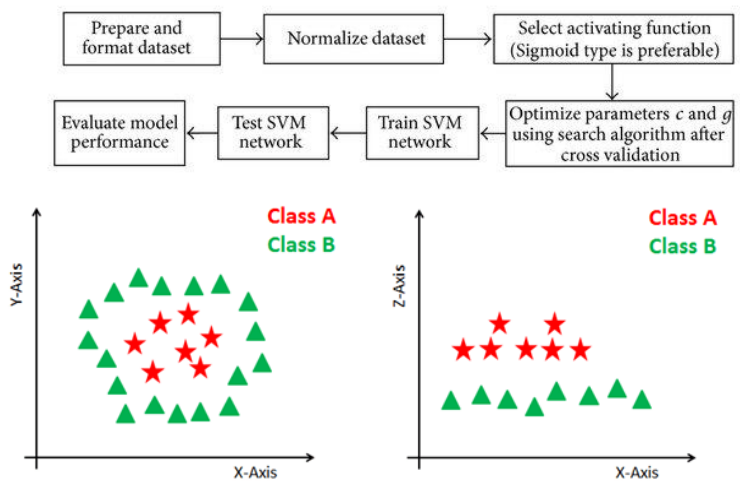

Fig. 1. General block diagram representation of SVM classifier and Representation of Maximum Marginal Hyperplane (MMH) 


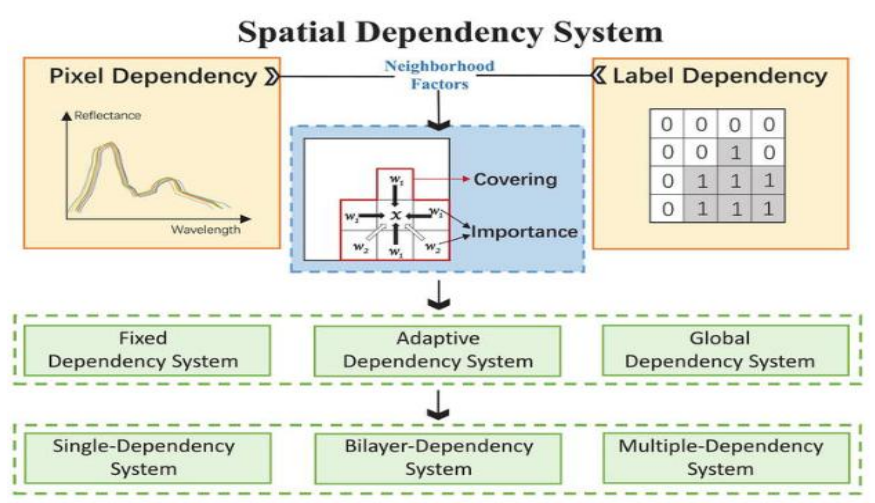

Fig. 2. Block Diagram Representation of Spatial dependency system used in Spectral-Spatial Classification Process (Source Intechopen)

2) Convolutional Neural Networks (CNN): The CNN is the novel 3-D based classification approach for HSI applies both spectral and spatial data. The method attained a decrease of the computation time and growing the precision in the classification of HSI than the conventional ANN techniques. The system lacked for multi-resolution. As to address the supervised and semi supervised approaches in classification, neural network based techniques have been proposed in the literature such as (i) Feed forward Neural network (FN) classifiers, used mainly to deal with secondorder optimization-based variations, (ii) Extreme Learning Machine (ELM). Later the concept of CNN was used in multi-hidden-layer networks. The architecture of deep CNN is shown in figure 6.

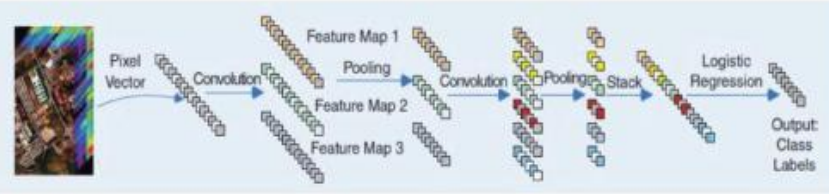

Fig. 3. Spectral Classification diagram based on Deep Convolutional Neural Network (Source Intechopen)

CNN was introduced to understand the deep representation of networks. The $\mathrm{CNN}$ is based on spectral signatures which are unique in nature. CNN classification approach resulted in a better performance compared to that of SVM. In CNN, during the learning process of unsupervised sparse features levels of spatial features are extracted. In case of deep $\mathrm{CNN}$ pixel-pair features were learned to achieve better classification.

\section{CHALLENGES AND DISCUSSION}

The main challenges faced by Hyperspectral imaging are curse of high dimensionality and accessing of the training samples. Some of the challenges are discussed below. The classification efficiency and kappa coefficients obtained using different classification approaches are also discussed.

\section{Case 1: SVM}

Dataset consists of 20 multispectral images of fruits where we used 4 types of classification of images using support vector machine.
The SVM classifier output showed the input image, preprocessed image and the identification of the image of fruits.

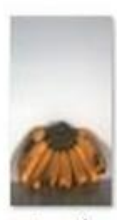

test1

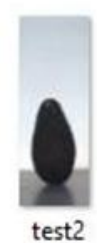

test2

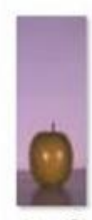

test3

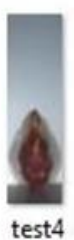

test4
Fig. 4. Test images of SVM classification

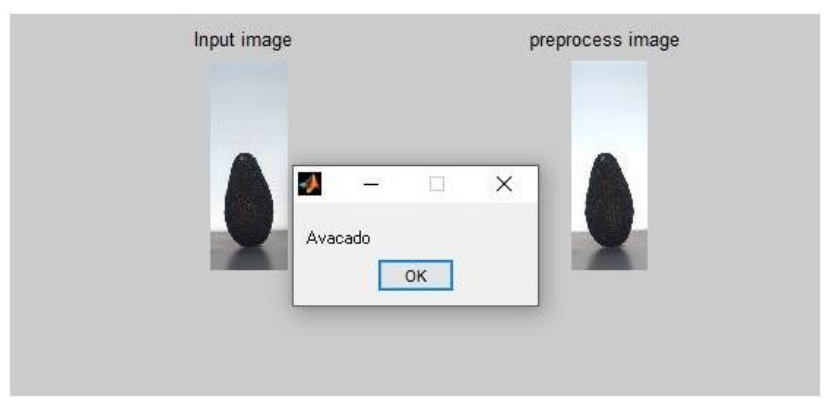

Fig. 5. SVM output at different stages in identification of fruits

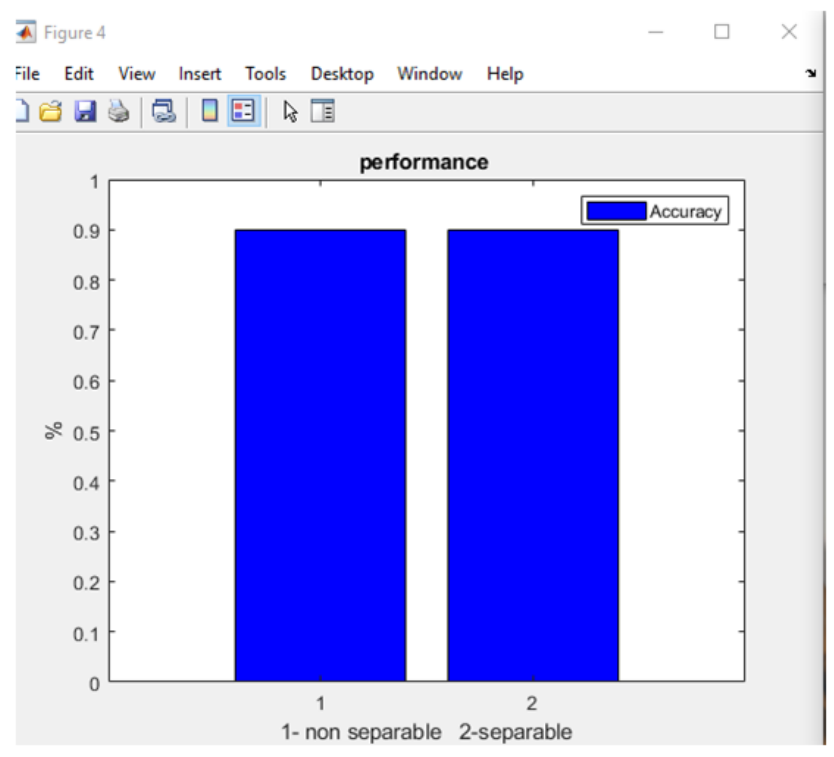

Fig. 6. SVM Accuracy plot

Case 2: KNN

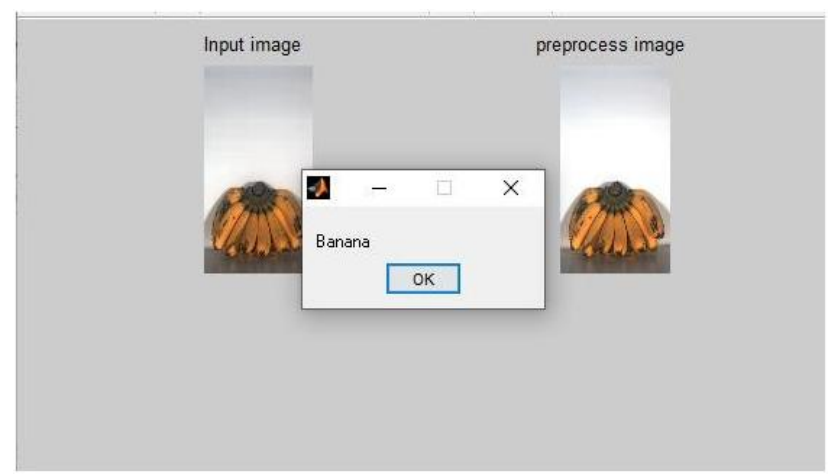

(a) 


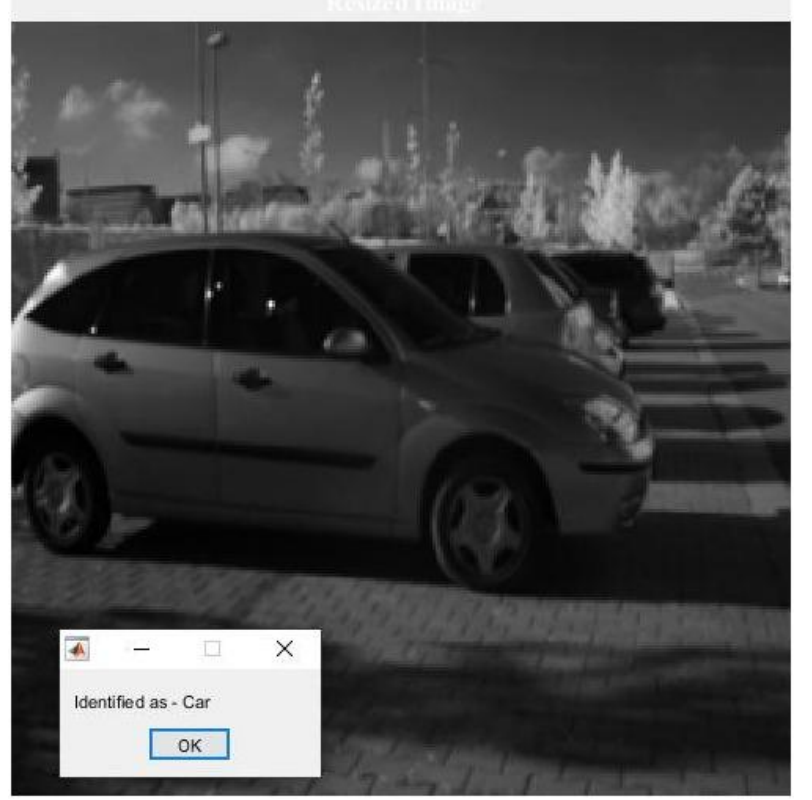

(b)

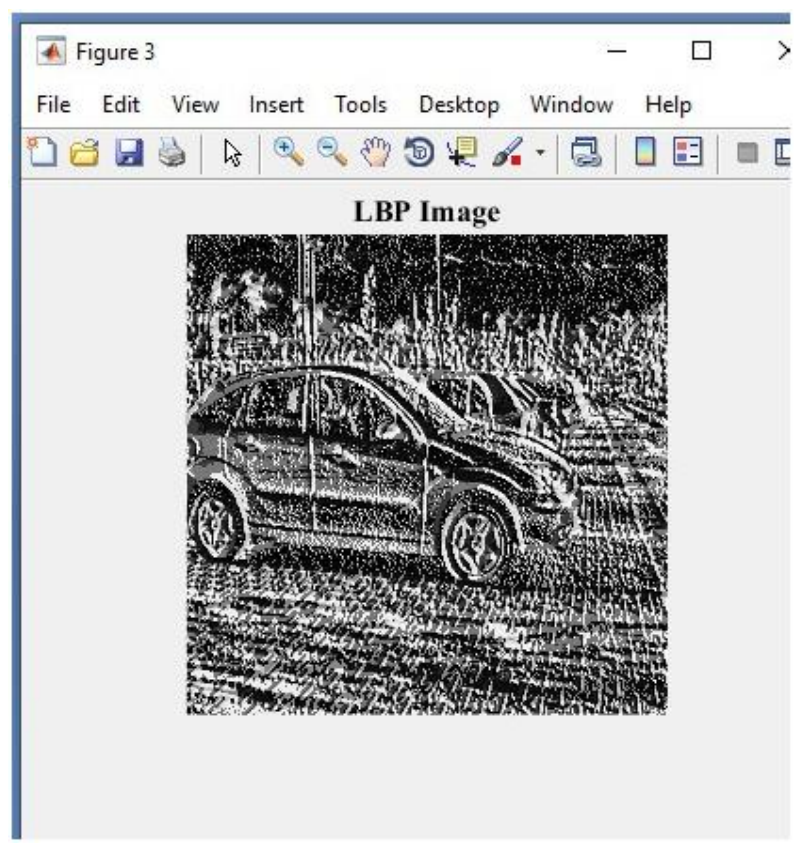

(c)

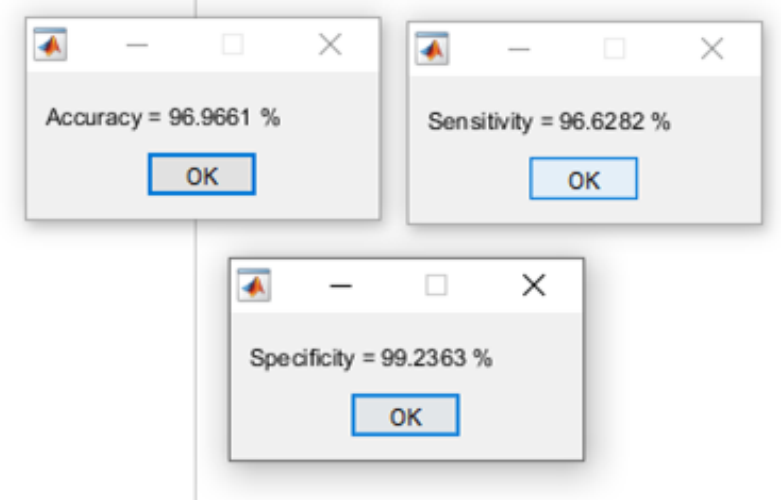

(d)

Fig. 7. (a) KNN Input image identification (b) Edge detected Image (c) Values of Accuracy, (d)Sensitivity and Specificity

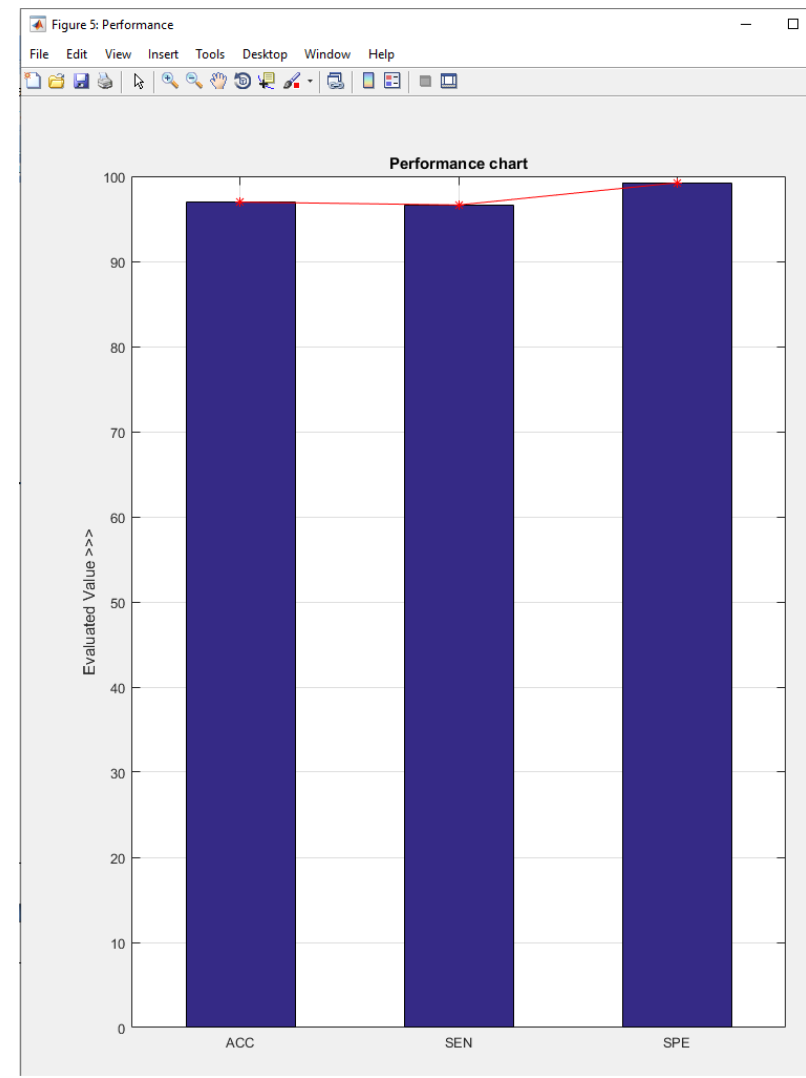

Fig. 8. Performance Chart

\section{CONCLUSION}

The comparison between SVM and $\mathrm{KNN}$ is presented in terms of their classification accuracy, Kappa and sensitivity. SVM provided an accuracy of 0.9 and KNN resulted in 0.96 . Hence it's found that KNN performed better in classification of multispectral imaging.

\section{REFERENCES}

[1] Rahul Nigam1, Rojalin Tripathy, Sujay Dutta, Nita Bhagia, Rohit Nagori, K. Chandrasekar, Rajsi Kot, Bimal K. Bhattacharya and Susan Ustin (2019) "Crop type discrimination and health assessment using Hyperspectral imaging" Journal of Current Science, Vol. 116, 10.

[2] Qureshi, R., Uzair, M., Khurshid, K., \& Yan, H. (2019), "Hyperspectral document image processing: Applications, challenges, and future prospects. Pattern Recognition", 90, 12-22.

[3] Zhang, L., Zhang, L., Du, B., You, J., \& Tao, D. (2019), "Hyperspectral Image Unsupervised Classification by Robust Manifold Matrix Factorization”, Information Sciences, 485, 154-169.

[4] Liu, Y., Wu, T., Yang, J., Tan, K., \& Wang, S (2019), "Hyperspectral band selection for soybean classification based on information measure in FRS theory, Bio- systems Engineering, 2019 178, 219-232.

[5] Zhou, F., Hang, R., Liu, Q., \& Yuan, X. (2019), "Hyperspectral image classification using spectral-spatial LSTMs", Neurocomputing, 328, $39-47$.

[6] Lan, R, Li, Z., Liu, Z., Gu, T., \& Luo, X. (2019), "Hyperspectral image classification using $\mathrm{k}$-sparse de- noising autoencoder and spectral-restricted spatial characteristics", Applied Soft Computing, 2019, pp 74, 693-708.

[7] Carlan, I, Mihai, B. A., Nistor, C., \& Grobe-Stoltenberg (2019), "Identifying urban vegetation stress factors based on open access remote sensing imagery and field observations", Ecological Informatics 55, pp. 01032.

[8] Hasan, M, Ullah, S., Khan, M. J., \& Khurshid, K (2019), "Comparative Analysis of SVM, ANN and CNN for Classifying Vegetation Species Using Hyperspectral Thermal Infrared Data", International Archives of the Photogrammetric, Remote Sensing \& Spatial Information Sciences. 
[9] Sun, Z., Zhao, X., Wu, M. and Wang, C (2019), "Extracting Urban Impervious Surface from WorldView-2 and Airborne LiDAR Data Using 3D Convolutional Neural Networks", Journal of the Indian Society of Remote Sensing, 47(3), pp.401-412.

[10] Brabant Ca, Alvarez-Vanhard E., Morin G., Thanh Nguyen K., Laribi A., Houet T (2018), "Evaluation of Dimensional Reduction Methods on Urban Vegetation Classification Performance using hyperspectral Data", IEEE, pp.1636-1639.

[11] Cao Jinging, Wanchun Leng, Kai Liu, Lin Liu, Zhi He ID and Yuanhui Zhu (2018), "Object-Based Mangrove Species Classification Using Unmanned Aerial Vehicle Hyperspectral Images and Digital Surface Models", Article published in Hyper Spectral Remote Sensing for Forest and Trees outside Forests.

[12] Peng, J, Jiang, X., Chen, N., \& Fu, H (2018), “Local Adaptive Joint Sparse representation for Hyperspectral image classification. Neuro computing", 2019 334, 239-248.
[13] Zhao, G., Liu, G., Fang, L., Tu, B., \& Ghamisi, P (2018), Multiple Convolutional Layers Fusion Framework for Hyperspectral Image Classification. Neurocomputing, 2019, pp 339, 149-160.

[14] Yinhua, X Gao, R Wei - Optik (2018), "Hyper spectral image classification based on adaptive segmentation", Optik Volume 172.

[15] Annala, L., Eskelinen, M., Hämäläinen, J., Riihinen, A. and Pölönen, I (2018), "Practical approach for Hyperspectral image processing in python", International Archives of the Photogrammetry, Remote Sensing and Spatial Information Sciences (Vol. 42, No.3), International Society for Photogrammetry and Remote Sensing.

[16] Brabant, C., Alvarez-Vanhard, E., Morin, G., Laribi, A. and Houet, T (2018), "Evaluation of Dimensional Reduction Methods on Urban Vegetation Classification Performance Using Hyperspectral Data", IGARSS 2018-2018 IEEE International Geosciences and Remote Sensing Symposium (pp. 1636-1639), IEEE. 